\title{
Evaluation of CERES-Rice model for the selected rice varieties of Kerala
}

\author{
ARJUN VYSAKH*, B. AJITHKUMAR and A. V. M. SUBBA RAO' \\ Dept. of Agri. Meteorology, Kerala Agricultural University, Thrissur. \\ ${ }^{1}$ Central Research Institute for Dry land Agriculture, Hyderabad. \\ Email:*arjunvysakh3@gmail.com
}

\begin{abstract}
A field experiment was conducted at Thrissur during kharif seasons of 2013 and 2014 using two rice varieties (Jyothi and Kanchana) and five dates of planting (June 5th, June 20th, July 5th, July 20th and August 5 th). CERES-Rice model of DSSAT v 4.6 was used to simulate the phenology and grain yield of rice. Calibration of genetic coefficients was done with 6000 iterations and then the model was validated. The model could simulate the phenology of both the rice varieties with RMSE ranging between 0.89 to 1.92 for physiological maturity. The model overestimated the grain yield of rice cultivars which need further refinement.
\end{abstract}

Keywords: Rice, CERES model, calibration, genetic coefficients, simulation,validation.

Rice is intimately involved in the culture as well as in the food ways and economy of Indians especially Keralites. Climate plays an important role in rice production. Rice is vulnerable to change in weather and its cultivation continues to be a risky enterprise under unfavourable environment, despite advances made in rice production technologies. Crop simulation models can be used as a tool for agricultural risk analysis. The CERES-Rice model is used to analyse the suitability of the rice cultivars and to determine the optimum planting date for rice in the central zone of Kerala. (Saseendran et al. 1998). Once a crop model has been validated, it can be used to match variety and site rather than carry out extend field experiments (Mankeb, 1993). According to Kumar et al. (2007) CERES-Rice model was able to predict phenological developments accurately. The CERES-Rice model can be used for decision making for crop management and improving the resource use efficiency (Singh et al., 2015). The present study was carried out to validate the CERES-Rice model of DSSAT v 4.6 for the rice varieties Jyothi and Kanchana in Kerala.

\section{MATERIALS AND METHODS}

The field experiments were conducted during the kharif seasons of 2013 and 2014 at the Agricultural Research Station, Mannuthy, Kerala Agricultural University, Thrissur.. The experiments were laid out in split plot design with five dates of planting at an interval of 15 days from June 5 th to August 5th as the main plot treatments and two varieties (Jyothi and Kanchana) as sub plot treatments with four replications. The recommended management practices were followed. The daily meteorological data recorded in the observatory was used.

CERES-Rice model of DSSAT v 4.6 was used to analyze the rice grain yield and phenology. All the input files required to run the model viz.weather file, soil file and crop file were created using records from the station. Accuracy in simulation of yield, phenology and growth requires the accurate genetic coefficients, which were determined by the 'Gencalc' (Genotype Coefficient Calculator) in the decision support system for agrotechnology transfer (DSSAT). A total of 6000 iterations were done to calibrate the genetic coefficient for each variety. These coefficients were adjusted until there was a close match between the observed and simulated dates of panicle initiation, anthesis, physiological maturity and grain yield (Table 1).

The validation of the model is the comparison of simulated values of model with the observed values from experiments. Root mean square error (RMSE) and D - stat (index of agreement) was used to evaluate the model performances.

$$
\begin{aligned}
& \text { RMSE }=\left[\sum_{i=1}^{n} \frac{(P i-O i)^{2}}{n}\right]^{0.5} \\
& \mathrm{D}-\text { stat }=1-\frac{\sum_{i=1}^{n}(P i-O i)^{2}}{\sum[(P i-\text { Oiavg })+(O i-\text { Oiavg })]^{2}}
\end{aligned}
$$

Where, RMSE is root mean square error and D-stat is index of agreement, $\mathrm{Oi}$ is Observed value, $\mathrm{Pi}$ is Predicted value, Oiavg is average of observed value and $\mathrm{n}$ is number of observations. The D-stat value should approach unity and the RMSE should approach zero for good performance of the model (Willmott, 1982). 
Table 1: Calibrated genetic coefficients for rice varieties, Jyothi and Kanchana

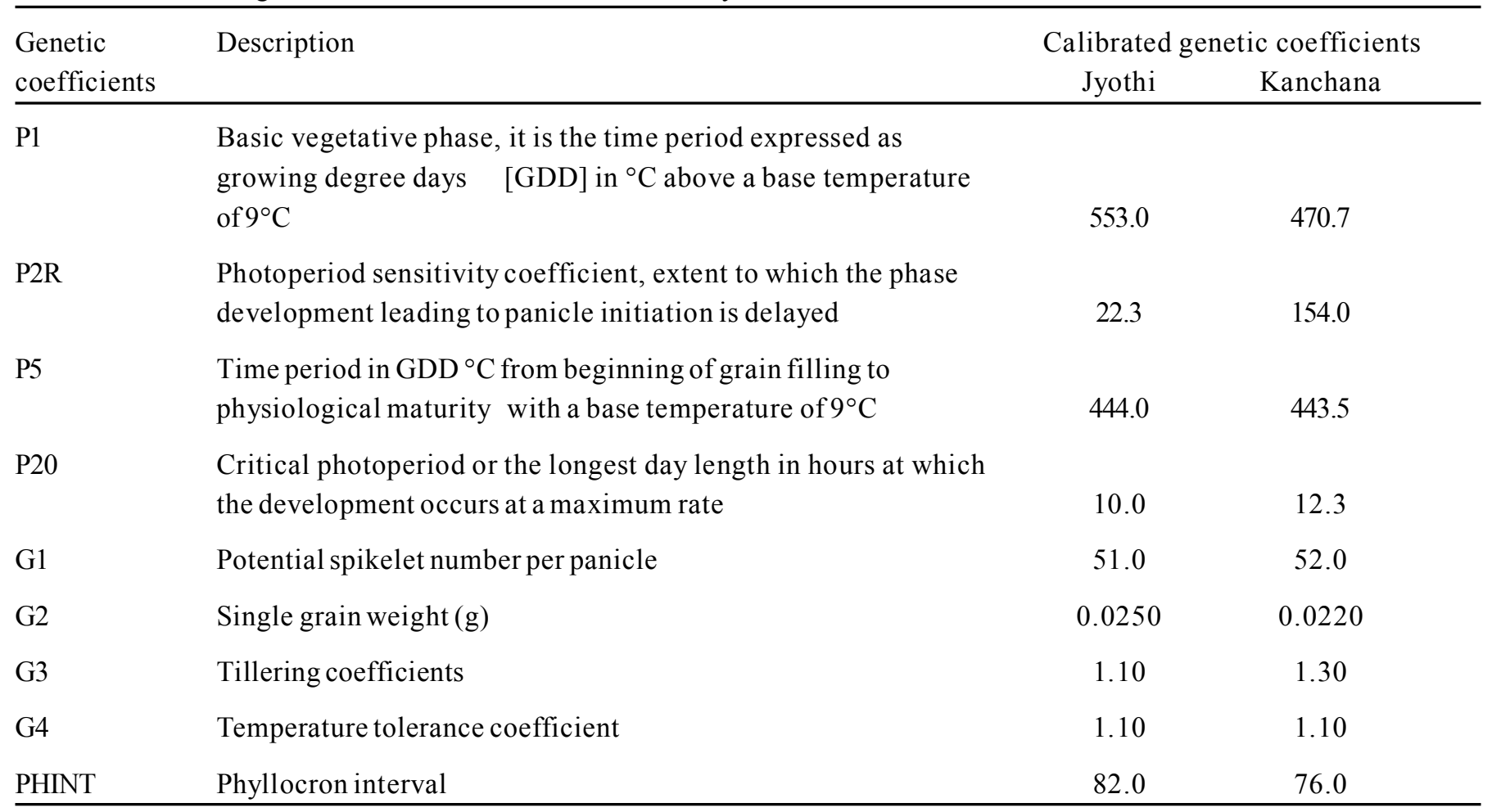
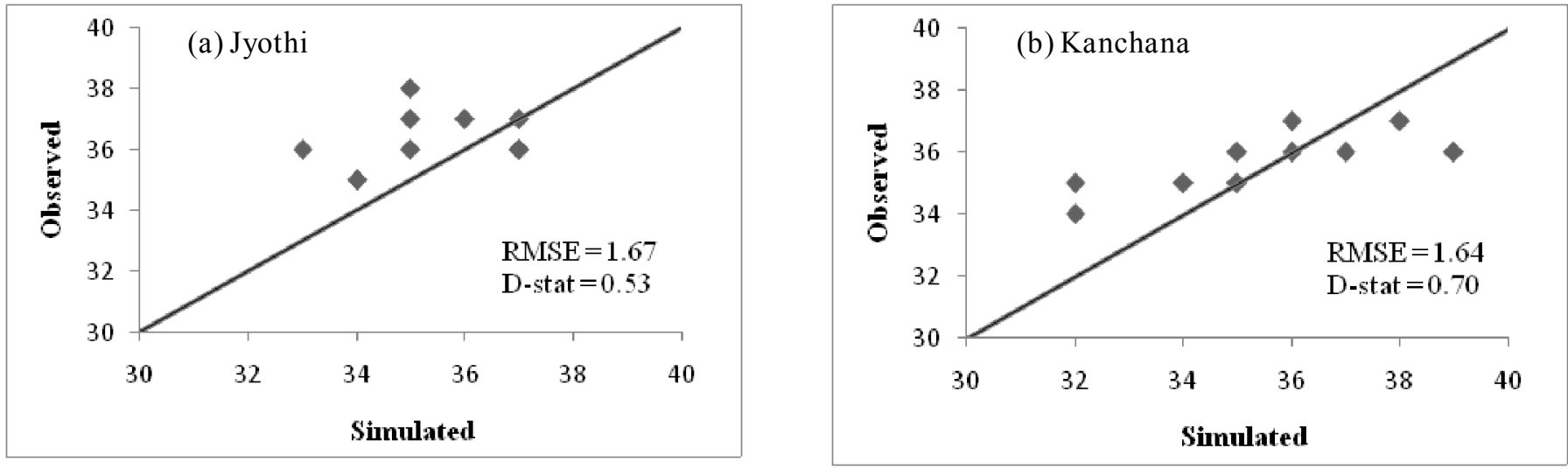

Fig. 1:Observed and simulated panicle initiation day
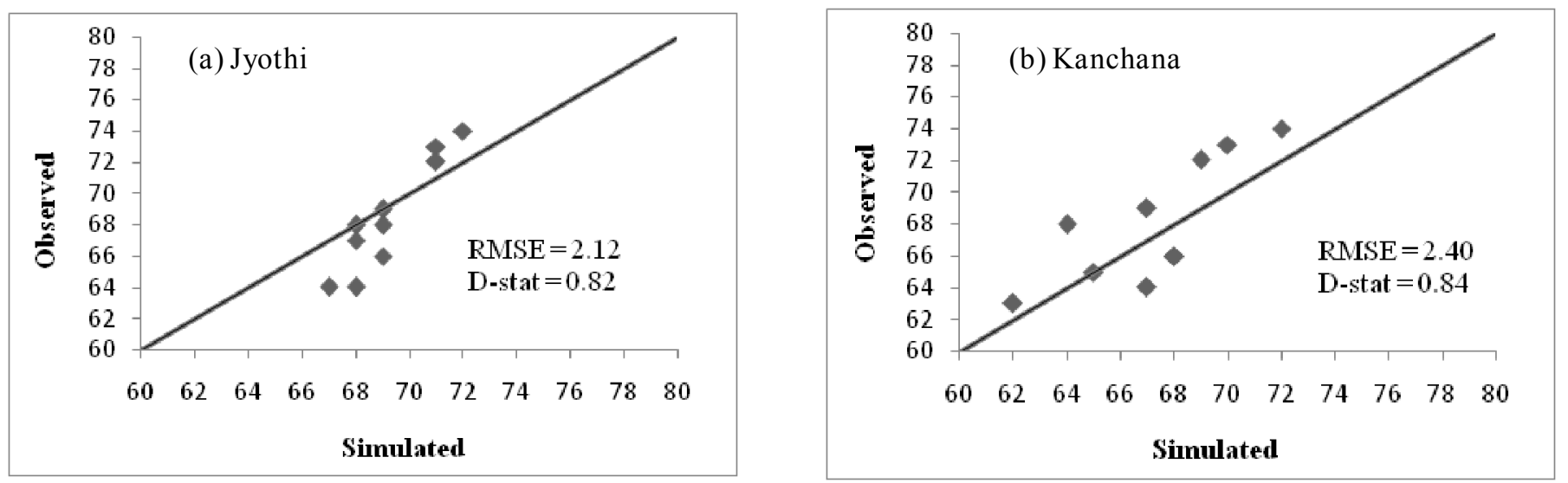

Fig. 2:Observed and simulated anthesis day 


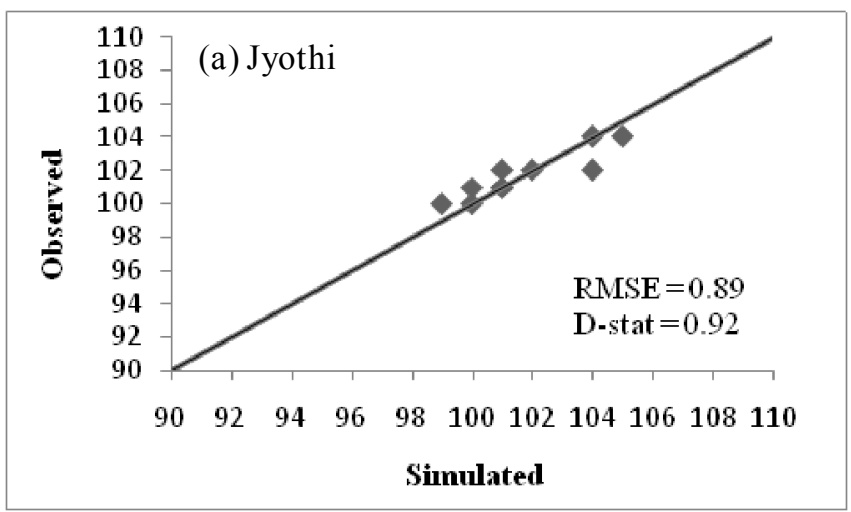

Fig. 3:Observed and simulated physiological maturity day

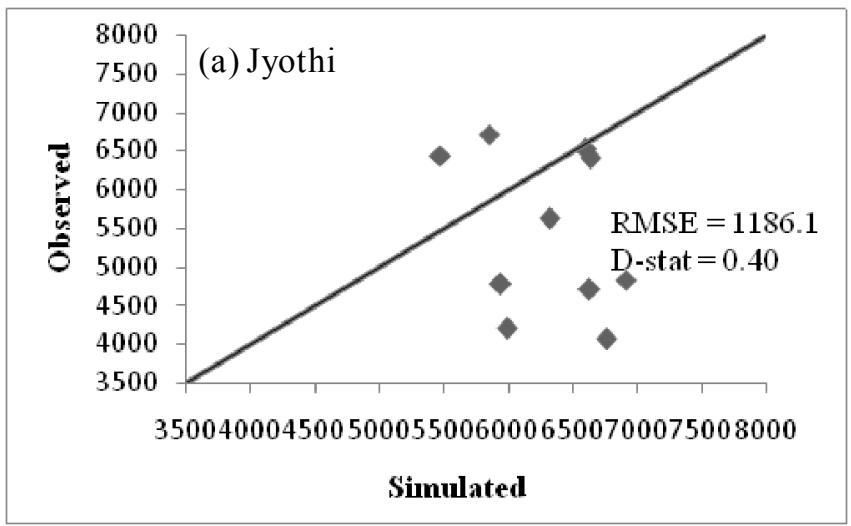

Fig. 4:Observed and simulated grain yield $\left(\mathrm{kg} \mathrm{ha}^{-1}\right)$

\section{RESULTS AND DISCUSSION}

The validation was done to assess the performance of the CERES-Rice model to predict the phenology and grain yield of the crop during the period of study.

\section{Phenology}

There was a good agreement between observed and simulated phenology for both Jyothi and Kanchana. The model could predict the panicle initiation day accurately, with percentage deviation within $10 \%$ in all plantings during both the years, with an RMSE of 1.64 and 1.67 and D-stat of 0.53 and 0.70 for two cultivars suggesting a good agreement (Fig. 1).

The model was able to simulate the anthesis day accurately with an RMSE of 2.1 and 2.4 D-stat of 0.82 and 0.84 for two cultivars (Fig.2). The percentage deviation between observed and predicted values was within $10 \%$ in all plantings of Jyothi and Kanchana, indicating good performance of the model.

The physiological maturity day was well predicted by the model compared to other phenological phases of both the varieties. The percentage deviation between
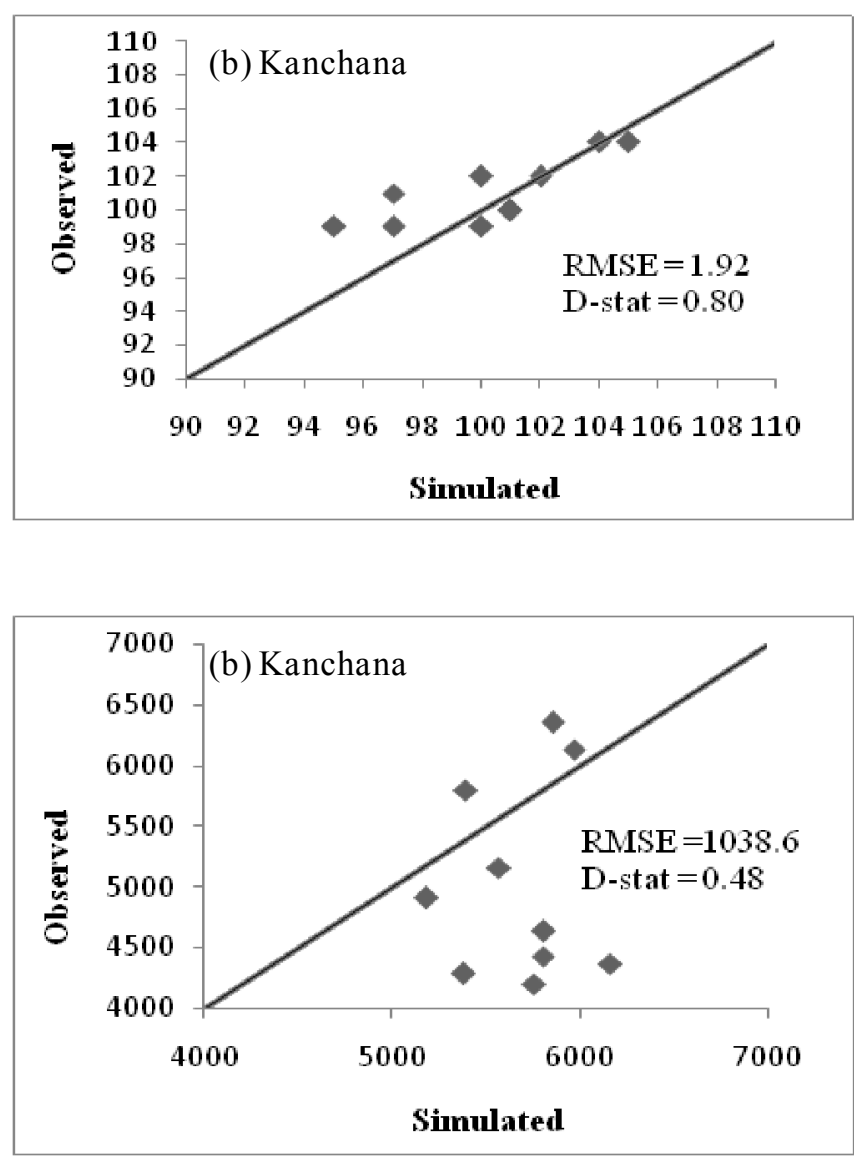

observed and predicted values was within $2 \%$ for all the plantings in both the varieties. The RMSE was 0.89 and 1.92 for two cultivars while D-stat was 0.92 and 0.80 (Fig.3).

\section{Grain yield}

The model generally overestimated the grain yield.The RMSE and D-stat for the yield of Jyothi were 1186.1 and 0.40 repectively (Fig. $4 a$ ). The predicted yields were closer to the observed values for early planted crops as compared to late planted crops during both the years with an RMSE of 1038.6 and D-stat of 0.48 (Fig.4b). The model also over estimated the yield of Kanchana.

\section{CONCLUSION}

The model could predict the phenophases more accurately. But the model over estimated the grain yield in many of the plantings. The variable performance of the model is probably due to a combination of deficiencies in model inputs, experimental observations, inclusion of nonmodelled factors (such as disease, pests, lodging etc.) in model validation. The CERES-Rice model needs further validation with more number of years of data in order to increase confidence level prior to the application of the model. 


\section{REFERENCES}

Kumar, Dalip, Herath, S., Saha, S. and Dash, R. N. (2007). CERES Rice model: Calibration, Evaluation and application for solar radiation stress assessment on rice production. J. Agrometerol., 9(2):138-148.

Mankeb, P. (1993). Calibration of genetic coefficients of paddy rice (Oryza sativa L.) for validation of the CERES-Rice model in northern Thailand. Available http:// mccweb.agri.cmu.ac.th/mccwwwthai/graduate/ thesis.prod03.html

Saseendran, S. A., Hubbard, K. G., Singh, K. K., Mendiratta, N.,
Rathore, L. S. and Singh, S. V. (1998). Optimum transplanting dates for rice in Kerala, India, determined using both CERES v3.0 and ClimProb. Agron. J., 90: 185-190.

Singh, P. K., Singh, K. K., Bhan, S. C., Baxla, A. K., Gupta, A., Balasubramanian, R. and Rathore,L. S. (2015). Potential yield and yield gap analysis of rice (Oryza sativa L.) in eastern and north eastern regions of India using CERESrice model. J. Agrometeorol., 17(2): 194-198.

Willmott, C. J. (1982). Some comments on the evaluation of model performance. Am. Meteorol.Soc. Bull.,63:13091313. 